\title{
Neuwahl par ordre de mufti: Bemerkungen zu den Urteilen des Landesverfassungsgerichts von Schleswig-Holstein vom 30. August 2010
}

von Hans H. Klein

\section{Analyse und Kritik der Entscheidungsgründe}

Die Entscheidungen des Landesverfassungsgerichts von Schleswig-Holstein (LVerfG) vom 30. August 2010 beruhen im Wesentlichen auf drei Aussagen. Zum einen wird die in Art. 10 Abs. 2 S. 2 der Landesverfassung auf 69 Mandate festgelegte Regelgröße des Landtags als Richtgröße verstanden, die nur ganz ausnahmsweise überschritten werden dürfe (dazu unter 1.). Zum anderen wird aus Art. 10 Abs. 2 S. 3 der Landesverfassung (LV), der gebietet, dass das Verfahren zur Wahl des Landtags ,,die Persönlichkeitswahl mit den Grundsätzen der Verhältniswahl“" zu verbinden hat, in Verbindung mit Art. 3 Abs. 1 LV (Wahlrechtsgleichheit) zwar prinzipiell zu Recht abgeleitet, dass das Landtagswahlrecht dem Grundsatz der Erfolgswertgleichheit Rechnung zu tragen habe. Diesem wird aber eine aus meiner Sicht so nicht tragfähige strikte Auslegung gegeben (dazu unter 2.). Schließlich fußt die Begründung der Entscheidungen auf der Behauptung, die seit dem Erlass des geltenden Wahlgesetzes eingetretene Veränderung der politischen Lage habe dazu geführt, dass die $\S \S 1$ Abs. $1 \mathrm{~S} .2$ und Abs. 2, 3 Abs. 5 und 16 dieses Gesetzes ,in ihrem Zusammenspiel“ gegen Art. 3 Abs. 1 in Verb. mit Art. 10 Abs. 2 LV verstießen (dazu unter 3.).

\section{1. a)}

In der Auslegung durch das LVerfG verpflichtet Art. 10 Abs. 2 LV den Gesetzgeber, ein Wahlrecht zu schaffen, das ,,in der politischen Realität“ die Entste-

In Reaktion auf Klagen der Landtagsfraktionen von Bündnis 90/Die Grünen und dem Südschleswigschen Wählerverband (SSW) gegen die derzeit gültige Regelung der Überhangmandate bei Landtagswahlen, erklärte das Schleswig-Holsteinische Landesverfassungsgericht im vergangenen Sommer Teile des Landtagswahlrechts für verfassungswidrig und ordnete eine Änderung des Wahlgesetzes bis zum 31. Mai 2011 sowie eine vorgezogene Landtagswal bis spätestens 30. September 2012 an. Der vorliegende Beitrag nimmt Bezug auf eine gutachtliche Äußerung, die der Verfasser im Auftrag der CDU-Fraktion des SchleswigHolsteinischen Landtags abgegeben hat. 
hung von Überhang- und Ausgleichsmandaten so weit wie möglich verhindert, um auf diese Weise zu gewährleisten, dass die Zahl von nicht mehr als 69 Abgeordneten möglichst eingehalten wird. ${ }^{2}$ Das Gebot der ,repräsentativen Wiedergabe des Wählerwillens“" stehe unter der Vorgabe, dass die Zahl von 69 Landtagsmandaten „möglichst erreicht, allenfalls geringfügig“ überschritten werde. ${ }^{3}$ Zwar ergäben ,die originären Gesetzesmaterialien“ nicht, dass der Verfassungsgeber eine zahlenmäßig verbindliche Zielvorgabe habe treffen wollen, sowohl der Wortlaut des Art. 10 Abs. 2 LV als auch die Debatten im Landtag zeigten aber, welche Bedeutung der Festlegung auf 69 Mandate beizumessen sei. ${ }^{4}$ Das wird in der Folge vor allem entstehungsgeschichtlich begründet. ${ }^{5}$ Dabei bleibt nicht unerwähnt, dass die SPD-Fraktion des Landtags in den Debatten zu Beginn der 1990er Jahre in der Zahl von (damals) 75 Abgeordneten nicht ein ,exaktes Ziel, sondern nur eine „Ausgangszahl“ sehen wollte, ${ }^{6}$ dass während der Debatten zur Verfassungsänderung von 2003 Einigkeit bestand, dass die Zahl von 69 Mandaten ,eine regulär vorgesehene Zahl“ und eine endgültige Größe des Landtags von höchstens 75 bis 77 Abgeordneten erstrebenswert sei. ${ }^{7}$

\section{1. b)}

Die Entstehungsgeschichte in der Darstellung des LVerfG lässt durchaus den Schluss zu, dass es dem Verfassungsgeber darum gegangen ist, die Zahl der Mandate zu begrenzen. Wichtiger aber war es ihm offenkundig, im Landtag den Wählerwillen repräsentativ abzubilden, also etwaige Überhangmandate einerseits in Kauf zu nehmen, andererseits jedoch weitgehend durch Ausgleichsmandate zu kompensieren. Der Wortlaut des Art. 10 Abs. 2 LV liefert keinen zwingenden Anhaltspunkt für die vom LVerfG behauptete Dominanz der in Satz 2 festgelegten Mandatszahl gegenüber dem in den Sätzen 4 und 5 genannten Ziel, Überhangmandate anzuerkennen und durch die Zuweisung von Ausgleichsmandaten dem Stimmenproporz zumindest weitgehend Rechnung zu tragen. Ein Rangverhältnis zwischen Art. 10 Abs. 2 S. 2 einerseits und Art. 10 Abs. 3 und 4 LV andererseits im Sinne einer Überordnung von Satz 2 über die folgenden Sätze mit

\footnotetext{
2 LVerfG 1/10, Rdnr. 82.

3 Ebd., Rdnr. 83.

4 Ebd., Rdnr. 84.

5 Ebd., Rdnrn. $86 \mathrm{ff}$.

6 Ebd., Rdnr. 87.

7 Ebd., Rdnr. 88.
} 
der Folge, dass ,,vorrangig (sic!) Überhangmandate zu vermeiden sind““, 8 ist nicht erkennbar. Aus der Abfolge der Sätze - Satz 2: Begrenzung der Mandatszahl, Sätze 3 und 4: Anerkennung von Überhangmandaten plus Zuteilung von Ausgleichsmandaten - ist vielmehr umgekehrt zu folgern, dass bei der Berechnung der Sitzzahl zwar von der Zahl von (mindestens) 69 Abgeordneten auszugehen ist, die tatsächliche sich aus der Wahl ergebende Mandatszahl aber unter Beachtung der Sätze 3 bis 5 des Art. 10 Abs. 2 LV zu berechnen ist: Art. 10 Abs. 2 Satz 2 gilt nach Maßgabe der Sätze 3 bis 5 dieser Vorschrift. Die Entstehungsgeschichte, selbst wenn die ihr vom LVerfG zugeschriebene Deutung zuträfe, ist für die Auslegung des Art. 10 Abs. 2 LV demgegenüber unerheblich, da der Wortlaut der Vorschrift einen Zweifel an der gleichen Gewichtung der in ihm enthaltenen Bestimmungen nicht zulässt. Das Bundesverfassungsgericht (BVerfG) hat jedenfalls immer wieder darauf hingewiesen, dass den Gesetzesmaterialien für die Auslegung von Verfassungsnormen eine ausschlaggebende Bedeutung in der Regel nicht zukommt. ${ }^{9}$

\section{2. a)}

Im Verhältniswahlsystem müssen die abgegebenen Stimmen nicht nur den gleichen Zählwert, sondern grundsätzlich auch den gleichen Erfolgswert haben. In Ansehung der Erfolgswertgleichheit hat die Rechtsprechung des BVerfG zwar einerseits den strengen und formalen Charakter dieses Grundsatzes betont, andererseits aber auch Differenzierungen zugelassen, soweit dafür ein ,zwingender Grund" besteht. ${ }^{10}$ Dies verlangt dem Gericht zufolge

,allerdings nicht, dass sich die Differenzierungen von Verfassungs wegen als zwangsläufig oder notwendig darstellen ... Es werden auch Gründe zugelassen, die durch die Verfassung legitimiert und von einem Gewicht sind, das der Wahlrechtsgleichheit die Waage halten kann. ... dabei ist es nicht erforderlich, dass die Verfassung diese Zwecke zu verwirklichen gebietet ... In diesem Zusammenhang rechtfertigt das Bundesverfassungsgericht Differenzierungen auch durch ,zureichende', , aus der Natur des Sachbereichs der Wahl der Volksvertretung sich ergebende Gründe' ... Hierzu zählt insbesondere die Verwirklichung der mit der Parlamentswahl verfolgten Ziele ...; dazu gehören die Sicherung des Charakters der Wahl als eines Integrati-

8 Ebd., Rdnr. 157.

9 Vgl. BVerfGE 111, 54 (91), mit weit. Nachw.

10 Zusammenfassend: BVerfGE 95, 408 (418 f.); BVerfG (Kammer), Beschluss vom 14. Februar 2005, NVwZ 2005, S. 568 (569), mit zahlr. Nachw. 
onsvorgangs bei der politischen Willensbildung des Volkes ... und die Gewährleistung der Funktionsfähigkeit der zu wählenden Volksvertretung ..."

Überdies fordert das BVerfG, dass

„,differenzierende Regelungen zur Verfolgung ihrer Zwecke geeignet und erforderlich sein ... (müssen). Ihr erlaubtes Ausmaß richtet sich daher auch danach, mit welcher Intensität in das - gleiche - Wahlrecht eingegriffen wird ... Ebenso können gefestigte Rechtsüberzeugung und Rechtspraxis Beachtung finden ... Der Gesetzgeber muss sich bei seiner Einschätzung und Bewertung nicht an abstrakt konstruierten Fallgestaltungen, sondern an der politischen Wirklichkeit orientieren“. ${ }^{11}$

Man wird diese Formulierungen dahin zu verstehen haben, dass sie den auch für die Anerkennung ,zwingender Gründe“ bestehenden, weit bemessenen Entscheidungsspielraum des Gesetzgebers umschreiben, den das BVerfG zu achten hat.

Im Blick auf diesen Maßstab hat das BVerfG etwa unterschiedliche Wahlkreisgrößen (innerhalb bestimmter Grenzen), Unterschriftenquoren, Sperrklauseln (bis zu einer Höhe von 5 Prozent), das Höchstzahlverfahren nach d'Hondt (das die stärkeren Parteien gegenüber den schwächeren leicht begünstigt - das LVerfG hingegen erklärt das d'Hondtsche Verfahren für ,,augenfällig problematisch“12), aber auch Überhangmandate (bis zu einer Höchstgrenze) verfassungsrechtlich nicht beanstandet.

Zu BVerfGE 95, 335, sei angemerkt, dass die Entscheidung zwar mit einem Stimmenverhältnis von 4:4 ergangen ist, die Meinung der die Entscheidung für die Zulässigkeit von Überhangmandaten tragenden vier Richter aber die Auffassung des BVerfG ist!

\section{2. b)}

Das LVerfG folgt dieser Meinung nicht. Es misst dem „Grundsatz der Erfolgswertgleichheit nicht nur eine begrenzte, sondern eine das einheitliche Wahlsystem insgesamt umfassende Bedeutung zu und folgert daraus - im Unterschied zum BVerfG -, ,,dass dem Gesetzgeber nur ein eingeschränkter Gestaltungsspielraum“ zusteht. ${ }^{13}$ Weil das Wahlsystem für den Schleswig-Holsteinischen Landtag als ,personalisierte Verhältniswahl“ zu charakterisieren sei, sei es von den Grundsätzen der Verhältniswahl „maßgeblich geprägt“ und also komme dem 
Grundsatz der Erfolgswertgleichheit „übergreifende Tragweite“ zu. ${ }^{14}$ Das schleswig-holsteinische Wahlsystem sei ein einheitliches Wahlsystem, ${ }^{15}$ durch Art. 10 Abs. 2 S. 5 LV werde die „Dominanz der Verhältniswahl“ bekräftigt. ${ }^{16}$ Die Pflicht zum Vollausgleich von Überhangmandaten - erstaunlicherweise erklärt das LVerfG an anderer Stelle, es könne dahinstehen, ob $§ 3$ Abs. 5 S. 3 LWahlG für sich genommen verfassungsgemäß sei ${ }^{17}$ - wird mit der These begründet, ,unter Rückgriff auf den strengen Grundsatz der Wahlgleichheit des Art. 3 Abs. 1 LV“ ergebe sich, ,dass nach der spezielleren Vorschrift des Art. 10 Abs. 2 S. 5 LV der Vollausgleich die Regel, der Teilausgleich eine zwingend begründungsbedürftige Ausnahme“ darstelle. ${ }^{18}$ Obgleich zunächst die Übereinstimmung der in Art. 3 Abs. 1 LV festgelegten Wahlgrundsätze mit denjenigen des Art. 38 Abs. 1 S. 1 GG betont wird, ${ }^{19}$ wird später behauptet, der auf dieser Grundlage dem Bundesgesetzgeber eingeräumte größere Gestaltungsspielraum bestehe nach schleswig-holsteinischem Verfassungsrecht nicht, denn Art. 3 Abs. 1 in Verb. mit Art. 10 Abs. 2 S. 5 LV verpflichte den Landesgesetzgeber, ,insgesamt auf den Proporz nach Zweitstimmen und auf einen Verhältnisausgleich, der grundsätzlich (?) auch die Mehrsitze deckt“.$^{20}$

\section{2. c)}

Die Argumentation des LVerfG kann nicht überzeugen. Die (zutreffende) Qualifizierung des für die Landtagswahl von Verfassungs wegen maßgebenden Wahlsystems als ,personalisierte Verhältniswahl“ lässt die vom Gericht gezogenen Schlussfolgerungen schon deshalb nicht zu, weil auch das Wahlrecht zum Deutschen Bundestag auf diesem Wahlsystem beruht, ohne dass sich daraus die Notwendigkeit eines (zumal vollständigen) Ausgleichs von Überhangmandaten ergäbe. Auch für das Bundeswahlrecht hat die Erfolgswertgleichheit ,übergreifende Tragweite“, aber die Rechtsprechung des BVerfG leitet daraus gerade nicht $\mathrm{ab}$, dass für Überhangmandate Ausgleichsmandate $\mathrm{zu}$ gewähren seien. Zwar unterscheidet sich die Rechtslage in Schleswig-Holstein von der des Bundes dadurch, dass das Landesverfassungsgesetz ein Wahlrecht vorschreibt, „das 
die Persönlichkeitswahl mit den Grundsätzen der Verhältniswahl verbindet", und dass das Wahlgesetz ,für den Fall des Entstehens von Überhangmandaten Ausgleichsmandate vorsehen muss“ (Art. 10 Abs. 2 LV). Dadurch wird dem Landesgesetzgeber, anders als dem Bundesgesetzgeber, zwar verwehrt, ein Mehrheitswahlsystem einzuführen, einen vollständigen Ausgleich von Überhangmandaten verlangt Art. 10 Abs. 2 S. 5 LV aber gerade nicht. Wenn es sich bei dieser Vorschrift um die gegenüber Art. 3 Abs. 1 LV ,speziellere“ Norm handelt, wie das LVerfG annimmt, ${ }^{21}$ dann ist es geradezu widersprüchlich, daraus zu schließen, der Vollausgleich sei von Verfassungs wegen die nahezu ausnahmslos geltende Regel. ${ }^{22}$

Die vom LVerfG verworfene Vorschrift des $\S 3$ Abs. 5 S. 3 LWahlG befindet sich in voller Übereinstimmung mit der bereits skizzierten Rechtsprechung des BVerfG. Das System der ,personalisierten Verhältniswahl“ beschreibt das BVerfG dahin, der Gesetzgeber (in Schleswig-Holstein: der Verfassungsgesetzgeber: Art. 10 Abs. 2 S. 3 LV) habe damit den Proporz nach Zweitstimmen nicht zum ausschließlichen Verteilungsmaßstab erhoben. Der Erfolgswertgleichheit der Stimmen komme in diesem Wahlsystem von vornherein nur eine begrenzte Tragweite zu, was durch das Ziel der vorgeschalteten Mehrheitswahl, eine engere Beziehung zwischen den Wählern und den gewählten Wahlkreiskandidaten herzustellen, gerechtfertigt sei. Überhangmandate seien also keineswegs nur im Rahmen der durch das mathematische Sitzverteilungsverfahren ohnehin unvermeidbaren Ungleichheiten zulässig. Die ex ante zu beurteilende Erfolgschancengleichheit aller Stimmen werde durch die Möglichkeit von Überhangmandaten nicht beeinträchtigt. Allerdings müsse sich im Hinblick auf den „Grundcharakter der Wahl als Verhältniswahl“, der nicht aufgehoben werden dürfe, die Zahl der Überhangmandate in Grenzen halten. ${ }^{23} \mathrm{Zu}$ der Systementscheidung für die personalisierte Verhältniswahl gehöre es danach auch, dass „,ür den Regelfall“ der Legitimationsvorgang der Wahl ,hälftig persönlichkeitsbezogen, hälftig parteibezogen stattfinden soll“. ${ }^{24}$ Das in der Erststimme selbständig zur Geltung kommende Element der Personenwahl darf nach Ansicht des BVerfG nicht in den „Grundcharakter des geltenden Wahlrechts als Verhältniswahl“ eingeschmolzen werden. Nichts anderes besagt Art. 10 Abs. 2 S. 3 LV, der deutlicher noch als $\S 1$

21 Ebd., Rdnr. 123.

22 Ebd.

23 Vgl. BVerfGE 95, 335 (356 f., 358).

24 Ebd., 365. 
Abs. 1 S. 2 BWahlG, der von einer ,,mit der Personenwahl verbundenen Verhältniswahl“ spricht, die „,mit den Grundsätzen der Verhältniswahl“ zu verbindende „Persönlichkeitswahl“ in den Vordergrund stellt. Wenn nach Art. 10 Abs. 2 S. 4 und $5 \mathrm{LV}$ neben den Überhang- auch Ausgleichsmandate vorzusehen sind, so bestätigt dies bei unbefangener Auslegung nur, dass der Landesverfassungsgeber vorweggenommen hat, was das BVerfG in seinem Urteil vom 10. April 1997 ausgesprochen hat, dass nämlich die Belassung von Überhangmandaten dann (und nur dann!) durchgreifenden verfassungsrechtlichen Bedenken unterliegt, wenn sie den Proporz in unverhältnismäßiger Weise sprengen würde. ${ }^{25}$ Dem entspricht es wiederum, dass $\S 3$ Abs. 5 S. 3 LWahlG (wie das LVerfG bestätigt hat) die ,weiteren Sitze“ auf das Doppelte der „Mehrsitze“ begrenzt und damit jedenfalls bisher - erreicht, dass die unausgeglichene Zahl der Überhangmandate jene Grenze nicht sprengt.

In diesem Zusammenhang fällt auf, dass das LVerfG dem $\S 3$ Abs. 5 S. 3 LWahlG lediglich die Funktion zuschreibt, nach Möglichkeit die (vermeintlich vorrangige) Zielvorgabe von 69 Mandaten einzuhalten. ${ }^{26}$ Ob die Vorschrift dieses Ziel überhaupt verfolgt, ist allerdings durchaus zweifelhaft. Entscheidend für die hier getroffene Regelung ist ersichtlich etwas ganz anderes: sie will - wie Art. 10 Abs. 2 S. 4 und 5 LV und $\S 3$ Abs. 5 S. 1 LWahlG - dem Charakter des schleswig-holsteinischen Wahlsystems als „Persönlichkeitswahl“ Rechnung tragen, die zwar mit den „Grundsätzen der Verhältniswahl“ zu verbinden ist, aber dadurch gerade nicht uneingeschränkt dem Proporz unterworfen wird. Die gegenteilige Behauptung des LVerfG - die Verzerrung der Erfolgswertgleichheit diene nicht der Prämierung und Stärkung der Personenwahl ${ }^{27}$ - ist aus der Luft gegriffen. Zu ihrer Begründung stellt das Gericht die durch nichts bewiesene Behauptung auf, bis zur Landtagswahl 2009 habe sich das Ziel, eine Prämie für die Aufstellung ,attraktiver und überzeugungskräftiger“" Wahlkreisbewerber durch die Parteien zu vergeben, ,tatsächlich ... noch nie realisiert“. ${ }^{28}$ In Ermangelung jeglichen empirischen Belegs ist diese Behauptung willkürlich und gegenüber den Mitgliedern des Landtags anmaßend.

25 BVerfGE 95, 335 (365 f.).

26 vgl. LVerfG 1/10, Rdnrn. 145, 154, 156.

27 Ebd., Rdnr. 145.

28 Ebd., Rdnr. 147. 


\section{3. a)}

Das LVerfG begründet die Verfassungswidrigkeit der von ihm beanstandeten Vorschriften des LWahlG nicht zuletzt damit, dass sich die tatsächlichen Verhältnisse seit der Entstehung dieses Gesetzes wesentlich geändert hätten. Dass sich der Verfassungsgeber im Rahmen der Beratungen zu Art. 10 LV zur Frage des Voll- oder Teilausgleichs nicht ausdrücklich geäußert habe, wird darauf zurückgeführt, dass mit der Entstehung von Überhangmandaten in nennenswertem Umfang ,damals“ (1990) noch nicht gerechnet wurde. ${ }^{29}$ Die Vorstellung, bei tatsächlicher Entstehung von Überhangmandaten werde die Gewährung von doppelt so vielen weiteren Sitzen nicht ausreichen, um einen vollständigen Ausgleich zu erreichen, habe zu diesem Zeitpunkt fern gelegen. ${ }^{30}$ Das Zusammenwirken der Regelungen der $\S \S 1$ Abs. 2 S. 2 und Abs. 2, 16 LWahlG ,,(Zweistimmenwahlrecht, Anzahl der Wahlkreise und direkt gewählten Abgeordneten, Abweichung der Bevölkerungszahl eines Wahlkreises von bis zu 25 v.H. von der durchschnittlichen Bevölkerungszahl der Wahlkreise)“ führe ,gegenwärtig in der politischen Realität eines Fünfparteiensystems - zuzüglich einer verfassungsfesten Sonderrolle des SSW ${ }^{31}$ - systemisch dazu, dass Überhang- (und Ausgleichs)mandate in einer Größenordnung entstehen können“, in deren Folge die ,,in Art.

29 Ebd., Rdnr. 116.

30 Ebd., Rdnr. 119.

31 Mit welcher Nonchalance das LVerfG Verfassungsauslegung betreibt, erhellt aus der Beiläufigkeit, mit der es - es handelt sich insoweit um ein gänzlich überflüssiges obiter dictum - von einer ,,verfassungsfesten“ Sonderrolle des SSW spricht, ohne auch nur den Versuch einer Begründung seiner Behauptung zu unternehmen. Das BVerfG hat es zwar für zulässig erklärt, dass - unter Durchbrechung des Grundsatzes der Erfolgswertgleichheit - Parteien nationaler Minderheiten von der allgemeinen Sperrklausel freigestellt werden (BVerfGE 4, $31 \mathrm{zu} \S 3$ Abs. 1 LWahlG Schl.-H.; 1, 208 (253); 6, 84 (97 ff.), zu $§ 6$ Abs. 4 S. 2 (jetzt: $§ 6$ Abs. 6 S. 2) BWahlG. Zum Ganzen auch W. Schreiber, BWahlG. Kommentar, 8. Aufl., 2009, § 6 Rdnrn. 47 ff.). Das BVerfG hat aber für $\S 3$ Abs. 1 S. 2 LWahlG ebenso klargestellt, dass die Eigenschaft einer politischen Partei als Vertretung einer nationalen Minderheit keine so wesentliche Verschiedenheit begründet, dass der Gesetzgeber sie bei der Gestaltung der Rechte der politischen Parteien im Wahlverfahren berücksichtigen müsste. „Mit Bezug auf die parlamentarische Repräsentation des als Einheit gedachten Staatsvolkes begründet die Eigenschaft als Partei einer nationalen Minderheit keine Verschiedenheit, die wesentlich ist, und die der Gesetzgeber daher bei der Gestaltung der Rechte der politischen Parteien im Wahlkampf berücksichtigen müsste" (BVerfGE 31 [Leitsatz 3, S. 42] - Hervorhebungen im Original). Dem folgt das Schrifttum: A. von Mutius, in: derselbe/Wuttke/Hübner, Kommentar zur Landesverfassung Schleswig-Holstein, 1995, Art. 3 Rdnr. 13; im Ergebnis ebenso: S. Riedinger, in: Caspar/Ewer/Nolte/Waack, Verfassung des Landes Schleswig-Holstein, 2006, Art. 5 Rdnr. 27). Die Rechtslage in Brandenburg entspricht derjenigen in Schleswig-Holstein: wahlgesetzliche Privilegierung ohne verfassungsrechtliche Verpflichtung - $\$ 3$ Abs. 1 S. 2 und 3 LWahlG, Art. 25 LV (dazu: M. Ernst, in: Lieber/Iwers/Ernst, Verfassung des Landes Brandenburg, 2003, Art. 25 unter 5.). Die Sächsische Verfassung schützt wie Art. 5 LV Schl.-H. die (sorbische) nationale Minderheit, eine wahlrechtliche Privilegierung genießt diese jedoch nicht. 
10 Abs. 2 S. 2 LV zum Ausdruck gebrachte Zielgröße von 69 Abgeordneten regelmäßig überschritten wird“" ${ }^{32}$ Auf der Grundlage der, wie zuvor (oben unter 1. b)) dargelegt, überaus problematischen Prämisse, dass Art. 10 Abs. 2 S. 2 ein Vorrang vor Art. 10 Abs. 2 S. 4 und 5 LV einzuräumen sei, wird also behauptet, die Ablösung des im Jahr 1990 bestehenden Vierparteiensystems (CDU, SPD, FDP, Grüne- plus SSW) durch ein Fünfparteiensystem (Die Linke) begründe die Verfassungswidrigkeit der genannten Bestimmungen.

\section{3. b)}

Dass Veränderungen der Parteienlandschaft wahlrechtliche Bestimmungen in einem neuen Licht erscheinen lassen können, ist eine grundsätzlich berechtigte Annahme. „Es kann sich die vom Wahlgesetzgeber vorausgesetzte tatsächliche oder normative Grundlage geändert oder die bei Erlass der Bestimmung getroffene Prognose als irrig erwiesen haben“. ${ }^{33}$ Bezogen auf das geltende Wahlrecht zum Deutschen Bundestag kann beispielsweise der Schrumpfungsprozess, dem die (bisher) großen Parteien gegenwärtig unterworfen sind, zu einer Zunahme von Überhangmandaten führen, die die vom BVerfG gezogenen Grenzen mit der Folge einer nicht mehr hinnehmbaren Differenzierung der Erfolgswertgleichheit der Stimmen sprengt. Darauf müsste der Wahlgesetzgeber reagieren. Das schleswig-holsteinische Wahlrecht schließt diese Konsequenz durch die Gewährung von Ausgleichsmandaten aber gerade aus, es sei denn, was bisher nicht der Fall ist, die in $\S 3$ Abs. 5 S. 3 LWahlG vorgesehene Begrenzung der ,weiteren Sitze“ auf das Doppelte der Mehrsitze lasse so viele Überhangmandate unausgeglichen, dass die tolerable Grenze überschritten wird. Die Entscheidung des LVerfG beruht also allein auf der - wie gezeigt: unhaltbaren - Annahme, dass die in Art. 10 Abs. 2 S. 2 LV genannte Zahl von 69 Mandaten als Richt- und nicht als Ausgangsgröße verstanden wird.

\section{4.}

Das Urteil des LVerfG in der Sache LVerfG 1/10 zeigt eine Reihe von Möglichkeiten auf, wie auf der Grundlage des geltenden Verfassungsrechts die (vermeintlichen) Mängel des LWahlG behoben werden können: Reduzierung der Zahl der Wahlkreise, ${ }^{34}$ Veränderung ihres Zuschnitts: maximale Abweichung der

33 BVerfG (Kammer), Beschluss vom 17. November 2004, NVwZ 2005, S. 205 (206), mit Nachw. aus der Senatsrechtsprechung.

34 LVerfG 1/10, Rdnr. 160. 
Wahlkreisgröße vom Durchschnitt in Höhe von 15 Prozent (statt bisher 25 Prozent) unter Einschluss der Möglichkeit, auf die Zahl der Wahlberechtigten statt auf die Bevölkerungszahl abzustellen; ${ }^{35}$ Rückkehr zum Einstimmenwahlrecht; ${ }^{36}$ Mehr- statt Einmandatswahlkreise. ${ }^{37}$ Das sog. Grabenwahlrecht wird vom Gericht nicht erwähnt, was darauf hindeuten könnte, dass es dieses Wahlsystem (m.E. zu Unrecht) mit Art. 10 Abs. 2 S. 3 LV für nicht vereinbar hält.

Bevor über Einzelheiten einer Novellierung des LWahlG gesprochen wird, empfiehlt es sich jedoch, die verfehlte Interpretation des Art. 10 Abs. 2 S. 2 LV durch das LVerfG zu korrigieren. Durch die Einfügung des Wortes „,mindestens“ in Art. 10 Abs. 2 S. 2 LV würde klargestellt, dass die Zahl von 69 Abgeordneten keine Richt- sondern eine Ausgangsgröße ist. Das ist auch politisch gut vertretbar, weil im Ländervergleich der Landtag von Schleswig-Holstein bei einer Größe von 69 Abgeordneten im Hinblick auf die Fläche und die Einwohnerzahl des Landes eher am unteren Rande der Skala rangiert.

Der Verabsolutierung des Grundsatzes der Erfolgswertgleichheit, die nach Auffassung des LVerfG den in aller Regel vollständigen Ausgleich etwaiger Überhangmandate verlangt, kann ebenfalls nur auf der Ebene des Verfassungsrechts entgegengetreten werden. Will man das, könnte Art. 10 Abs. 2 um den folgenden Satz 6 ergänzt werden: „Die Ausgleichsmandate werden bis zur Höhe von 80 v.H. der entstandenen Überhangmandate im Verhältnis der von ihnen errungenen Stimmen an diejenigen im Landtag vertretenen Parteien vergeben, die keine Überhangmandate errungen haben." Mit dieser Formulierung würde auch eine so starke Aufblähung des Landtags vermieden, wie sie jetzt durch die in $\S 3$ Abs. 5 S. 3 LWahlG vorgeschriebene Berechnungsmethode bewirkt wird.

Würde die Landesverfassung in beiden Punkten geändert, könnte es an sich bei der bisherigen Fassung des LWahlG bewenden, denn das LVerfG hat die im Tenor genannten Vorschriften ja nicht für nichtig, sondern für unvereinbar mit der Verfassung erklärt. ${ }^{38}$ Es dürfte sich gleichwohl empfehlen, diese Vorschriften erneut in Gesetzesform zu beschließen. Würde nur Art. 10 Abs. 2 S. 2 LV geändert, muss in der Neufassung des LWahlG der Entscheidung des LVerfG Rechnung getragen werden, die, von nicht eindeutig definierten Ausnahmefällen abgesehen (das Gericht mag an unvermeidliche Konsequenzen bestimmter ma- 
thematischer Sitzverteilungsverfahren gedacht haben), mit Rücksicht auf den Grundsatz der Erfolgswertgleichheit der Stimmen einen vollen Ausgleich der Überhangmandate verlangt, deren Entstehung wohl nur dann mit an Sicherheit grenzender Wahrscheinlichkeit ausgeschlossen werden könnte, wenn die Zahl der Wahlkreise auf deutlich weniger als die Hälfte der Regelgröße von 69 Mandaten abgesenkt würde. Will man das nicht, bleibt nur, $\S 3$ Abs. 5 S. 3 LWahlG zu streichen.

\section{Anordnung von Neuwahl und Rechtsfolgen}

1.

In seiner Entscheidung in der Sache LVerfG 3/09 hat sich das LVerfG darauf beschränkt, die Unvereinbarkeit der $\S \S 1$ Abs. 1 S. 2 und Abs. 2, 3 Abs. 5 und 16 LWahlG mit Art. 3 Abs. 1 in Verb. mit Art. 10 Abs. 2 LV festzustellen. Im Verfahren LVerfG 1/10 wird der Gesetzgeber verpflichtet, spätestens bis zum 31. Mai 2011 eine mit der Landesverfassung übereinstimmende Rechtslage herbeizuführen, was, wie zuvor (unter I.4.) gezeigt, sowohl durch eine Änderung der Verfassung wie des einfachen Rechts geschehen kann. Außerdem wird angeordnet, bis zum 30. September 2012 eine Neuwahl des Landtags herbeizuführen.

\section{2.}

Die Beauftragung des Gesetzgebers, innerhalb einer bestimmten Frist eine verfassungskonforme Rechtslage herbeizuführen, bewegt sich auf der Linie der Rechtsprechung des BVerfG. ${ }^{39}$ Die Rechtsgrundlage für diese Vorgehensweise wird zwar nicht genannt (auch das BVerfG pflegt sie offen zu lassen), sie findet sich indessen in $\S 34$ LVerfGG, der $\S 35$ BVerfGG entspricht. Dieser Rechtsprechung liegt ein Vollstreckungsbegriff zugrunde, demzufolge unter Vollstreckung „,der Inbegriff aller Maßnahmen, die erforderlich sind, um solche Tatsachen zu schaffen, wie sie zur Verwirklichung des vom Bundesverfassungsgericht gefundenen Rechts notwendig sind“, zu verstehen ist. ${ }^{40}$

\section{3. a)}

Für das verfassungsgerichtliche Wahlprüfungsverfahren wird in $\S 50$ Abs. 1 LVerfGG auf das LWahlG verwiesen. Dieses behandelt die Wahlprüfung in den 
$\S \S 43$ ff. In $\S 46$ LWahlG wird der Fall geregelt, dass bei der Vorbereitung der Wahl oder bei der Wahl selbst Unregelmäßigkeiten vorgekommen sind, von denen anzunehmen ist, dass sie auf das Ergebnis der Landtagswahl von Einfluss gewesen sind. Erweist sich diese Annahme als begründet, ist eine Wiederholungswahl anzuordnen. Das LVerfG hat zu Recht festgestellt, dass eine Wiederholungswahl nicht in Betracht kommt, weil die von $\$ 46 \mathrm{LWahlG}$ vorausgesetzte Unregelmäßigkeit der Wahl hier nicht auf einer fehlerhaften Anwendung der Wahlvorschriften, sondern darauf beruht, dass die zu korrigierende UnregelmäBigkeit in der Verfassungswidrigkeit des Wahlgesetzes liegt. ${ }^{41}$ In diesem Fall eröffnet das - in Schleswig-Holstein wie im Bund nur bruchstückhaft geregelte Wahlfehlerfolgenrecht grundsätzlich die Möglichkeit, die Wahl für ungültig zu erklären. Diesen Weg ist das LVerfG wohlweislich nicht gegangen, da sonst die Neuwahl wiederum auf der Grundlage des in Teilen für verfassungswidrig befundenen Wahlgesetzes hätte durchgeführt werden müssen. ${ }^{42}$ Das BVerfG hat in vergleichbaren Fällen von einer Ungültigerklärung der Wahl abgesehen und sich damit begnügt, dem Gesetzgeber aufzugeben, den Wahlfehler bis zur nächsten oder gar übernächsten Wahl zu beheben. ${ }^{43}$

\section{3. b)}

Das LVerfG beschreitet einen anderen Weg: es ordnet die Durchführung einer Neuwahl des Landtags ,spätestens bis zum 30. September 2012“ und damit eine vorzeitige Beendigung der Wahlperiode an. Zur Begründung führt das Gericht im Wesentlichen aus: Es gelte das Gebot des geringstmöglichen Eingriffs. Eine Ungültigkeitserklärung setze, in den Worten des Bundesverfassungsgerichts einen erheblichen Wahlfehler von solchem Gewicht voraus, dass ein Fortbestand der in dieser Weise gewählten Volksvertretung unerträglich erscheine“.44 Der Landtagswahl von 2009 lägen Wahlfehler zugrunde, ,dass ihr insgesamt die verfassungsmäßige Grundlage entzogen ist“. Denn einerseits verfehle die Tatsache, dass der Landtag aus 95 Abgeordneten bestehe, die von der Verfassung vorgegebene Größe von 69 Mandaten in einem nicht mehr hinnehmbaren Ausmaß; andererseits führe die Begrenzung des Mehrsitzausgleichs zu einer nicht mehr zu rechtfertigenden Verzerrung des Wählerwillens. ${ }^{45}$ Diese Mängel seien 
so gewichtig, dass sich der Fortbestand des in verfassungswidriger Weise zusammengesetzten Landtags für die Dauer von weiteren vier Jahren, also bis zum regulären Ende der Wahlperiode, gegenüber dem hohen Verfassungsgut seiner rechtmäßigen Zusammensetzung nicht rechtfertigen lasse. ${ }^{46}$ Dem Landtag sei deshalb nur ein vorübergehender Bestandsschutz zuzugestehen. ${ }^{47}$ Gegenüber der eigentlich gebotenen Ungültigkeitserklärung sei als geringerer Eingriff in den Bestand des Landtags die Legislaturperiode auf den 30. September 2012 zu beschränken. $^{48}$

\section{3. c)}

Die vorzeitige Beendigung der Wahlperiode bei Gewährung eines nur vorübergehenden Bestandsschutzes ist dem Wahlfehlerfolgenrecht bisher fremd. Das BVerfG hat sich - bei durchaus vergleichbar gewichtigen Wahlmängeln - bisher stets dazu bekannt, dass der einmal gewählten Volksvertretung ,größtmöglicher Bestandsschutz“ zu gewähren sei. ${ }^{49}$ Dass der Bestandsschutz des Parlaments in der Rechtsprechung des BVerfG einen so hohen Rang genießt, folgt aus dem Demokratieprinzip. Denn das Parlament soll ,durch die Wahlprüfung in der Wahrnehmung seiner Aufgaben, insbesondere der Gesetzgebung und der Kontrolle der - von ihm als funktionsfähiges Organ erst hervorzubringenden - Regierung möglichst nicht beeinträchtigt werden“. ${ }^{50}$ In der Tat hat ja das Volk seinen Willen mit der Wahl bereits artikuliert. Es besteht ein fundamentales Interesse daran, diesem Willen für die Dauer der Wahlperiode Respekt zu zollen und so dem Gebot fristgemäßer Mandatserneuerung Rechnung zu tragen. Das Volk hat einen Anspruch darauf, dass die Volksvertretung in den von der Verfassung festgelegten zeitlichen Abständen, also ,in einer bestimmten historischen und nicht wiederholbaren Situation“", ${ }^{51}$ neu gewählt wird, der dann zum Ausdruck gebrachte Wählerwille für diese Zeitspanne Bestand hat und das Parlament innerhalb derselben seine Funktionen unbehindert erfüllen kann. Jeder wahlprüfungsrechtliche Eingriff in den Bestand des einmal gewählten Parlaments berührt diesen Anspruch des Wählers darauf, dass seine Stimme Geltung hat. Das

49 Vgl. BVerfGE 89, 243 (259); 103, 111 (134 f.); s. auch BVerfGE 121, 266 (311 ff.) und H.H. Klein, in Maunz/Dürig, Grundgesetz. Kommentar, Art. 41 Rdnrn. 105 ff.).

50 BVerfGE 103, 111 (134).

51 Koch, T.: Bestandsschutz für Parlamente - Überlegungen zur Wahlfehlerfolgenlehre, in: DVB1., 2000, 1093 (1098). 
LVerfG nimmt, obschon es sich (insoweit zu Unrecht) auf die Rechtsprechung des BVerfG beruft, eine andere Gewichtung vor, indem es sich mit erstaunlicher Leichtfüßigkeit über diese Erwägungen hinwegsetzt, zum Teil ohne sie einer Erwähnung auch nur für wert zu halten.

Wenig einleuchtend ist es auch, dass das Gericht - zudem auf der Grundlage überaus angreifbarer materiellrechtlicher Erwägungen (siehe oben I.) - die aus der Wahl 2009 hervorgegangene Zusammensetzung des Landtags einerseits als „unerträglich“ einstuft, die Volksvertretung andererseits aber längstens bis zur konstituierenden Sitzung des ,,spätestens am 30. September 2012“ neu zu wählenden Landtags bestehen lässt. Immerhin bleibt der Landtag danach für eine Dauer von mehr als der Hälfte des Restes der laufenden Wahlperiode ,im Amt“! Man hätte wohl auch erwarten dürfen, dass sich das LVerfG die Mühe macht, die „Erfindung“ der dem Wahlprüfungsrecht bisher unbekannten Rechtsfolge einer Verkürzung der Wahlperiode im Näheren zu begründen, zumal das geschriebene Wahlprüfungsrecht auch des Landes Schleswig-Holstein neben der Berichtigung des Wahlergebnisses ( $\$ 47$ LWahlG) ausschließlich die Wahlfehlerfolge der Ungültigkeit der Wahl kennt ( $\$ 46 \mathrm{LWahlG).} \mathrm{Bei} \mathrm{diesem} \mathrm{Befund} \mathrm{liegt} \mathrm{der} \mathrm{Vor-}$ halt nicht fern, das LVerfG habe die Bindung des Richters an das Gesetz (Art. 20 Abs. 3, 97 Abs. 1 GG, Art. 43 Abs. 1 LV), einen zentralen Ausdruck des Gewaltenteilungsprinzips, ${ }^{52}$ gröblich missachtet. ${ }^{53}$

\section{4.}

Die Entscheidungen des LVerfG binden die Verfassungsorgane des Landes $(§ 29$ LVerfGG) - auch wenn sie falsch sind. ${ }^{54}$ Zwar kann man die Frage aufwerfen, ob die Bindungswirkung entfällt, wenn das VerfG eine Entscheidung trifft, zu der es (wie vorliegend anzunehmen ist) nicht befugt ist. ${ }^{55}$ Ist aber, wie hier, ein Rechtsweg nicht gegeben (eine Möglichkeit, das BVerfG anzurufen, ist nicht ersichtlich), so liefe die Nichtanerkennung der Bindungswirkung auf einen politischen (Verfassungs-)Konflikt hinaus, in den einzutreten nur im äußersten Falle ratsam ist. Dem Landtag wird also nur übrig bleiben, sich den Entscheidungen des LVerfG zu beugen.

52 Vgl. etwa Hillgruber, C., in: Maunz, Th./Dürig, G.: Grundgesetz. Kommentar, Art. 97, Rdnr. 27.

53 Vgl. u.a. Krause, J.: Verfassungsbruch, in: Schleswig-Holsteinische Landeszeitung, 31.08.2010, 5.

54 Vgl. etwa Bethge, H., in: Maunz, Th./Schmidt-Bleibtreu, B./Klein, F./Bethge, H.: Bundesverfassungsgerichtsgesetz. Kommentar, § 31, Rdnrn. 10 ff. mit Nachw.

55 Vgl. Benda, E./ Klein, E.: Verfassungsprozessrecht, 2. Aufl., 2001, Rdnr. 1280. 


\section{5.}

$\mathrm{Zu}$ der Frage, wie die Legislaturperiode des Landtags zu einem Ende gebracht werden soll, verhält sich das LVerfG nicht. Es begnügt sich mit der Anordnung, spätestens bis zum 30. September 2012 eine Neuwahl herbeizuführen, ohne selbst die Beendigung der Wahlperiode zu diesem oder einem anderen Zeitpunkt zu verfügen.

\section{5. a)}

Nach der Landesverfassung kann die Legislaturperiode des Landtags auf zwei Wegen zu einem vorzeitigen Ende gebracht werden: durch Selbstauflösung nach Art. 13 Abs. 2 und durch Auflösung durch den Ministerpräsidenten nach einer gescheiterten Vertrauensfrage gemäß Art. 36 Abs. 1 LV. Beide Wege stehen offen. Allerdings ist weder der Ministerpräsident verfassungsrechtlich verpflichtet, die Vertrauensfrage zu stellen, um über ein negatives Vertrauensvotum die Befugnis zur vorzeitigen Auflösung des Parlaments zu erlangen - auch von dieser Befugnis Gebrauch zu machen, ist er keineswegs verpflichtet -, noch sind kraft ihres freien Mandats (Art. 11 LV) die Mitglieder des Landtags verpflichtet, dem Ministerpräsidenten ihr Vertrauen zu versagen oder für die Auflösung des Landtags zu stimmen. Die sich aus § 29 Abs. 1 LVerfGG ergebende Bindung von Landtag und Landesregierung an die Entscheidung des LVerfG in der Sache LVerfG 1/10 kann, zumal eine klare Äußerung des Gerichts dazu fehlt, schwerlich dahin verstanden werden, dass der Ministerpräsident oder die Mitglieder des Landtags gehalten sind, Entscheidungen zu treffen, die die Verfassung ausdrücklich ihrem freien Willen anheim gibt. Auch der (eher diffuse) ungeschriebene Grundsatz der Organtreue ${ }^{56}$ führt zu keinem anderen Ergebnis: er verpflichtet die Verfassungsorgane zu gegenseitiger Rücksichtnahme, aber nicht Abgeordnete zu einem bestimmten Gebrauch ihres Stimmrechts.

\section{5. b)}

Nach § 4 LWahlG bestimmt die Landesregierung den Wahltag. Das kann sie allerdings nicht nach Belieben tun, sondern nur in den vom Gesetz vorgesehenen Fällen: im Vorfeld des regulären Endes der Wahlperiode (Art. 13 Abs. 1 LV), bei vorzeitiger Beendigung der Wahlperiode (Art. 13 Abs. 2 und 3, 36 Abs. 1 LV) und im Falle einer Wiederholungswahl ( $\$ 46$ LWahlG). Zu erwägen ist, ob die Landesregierung durch das Urteil des LVerfG als ermächtigt (und verpflichtet) 
anzusehen ist, von ihrer Befugnis nach $\S 4 \mathrm{LWahlG}$ etwa in Analogie zu den in der Landesverfassung vorgesehenen Fällen der vorzeitigen Beendigung der Wahlperiode Gebrauch zu machen - nachdem Landesverfassung und Landeswahlgesetz vom Gesetzgeber in Übereinstimmung gebracht worden sind, unter Beachtung der in Art. 13 Abs. 3 LV vorgeschriebenen 70-Tage-Frist und Festsetzung eines Neuwahltermins spätestens bis zum 30. September 2012. Da das LVerfG in Anwendung des $§ 34$ LVerfGG eine entsprechende Vollzugsanordnung hätte treffen können, dies aber nicht getan hat, ist für die sei es unmittelbare, sei es analoge Anwendung des $\S 4$ LWahlG kein Raum. Die Analogie scheitert schon daran, dass es an einer ,planwidrigen Regelungslücke“ fehlt: der Gesetzgeber hat mit $§ 34$ LVerfGG für den vorliegenden Fall vorgesorgt. Da die Landesregierung nur innerhalb der (verfassungs)gesetzlich vorgegebenen Grenzen von ihrer Befugnis nach $\S 4$ LWahlG Gebrauch machen darf, sind ihr die Hände gebunden.

\section{5. c)}

In Betracht kommt weiterhin (vgl. Art. 59 f. LV) eine Verkürzung der Wahlperiode durch verfassungsänderndes Gesetz. ${ }^{57}$ Dieser Weg bietet gegenüber der Selbstauflösung nach Art. 13 Abs. 2 LV den Vorteil, dass er einen längeren Vorlauf bis zur Wahl erlaubt. ${ }^{58}$ Verfassungsändernde Gesetze bedürfen - wie die Selbstauflösung nach Art. 13 Abs. 2 LV - der Zustimmung von zwei Dritteln der Mitglieder des Landtags (Art. 40 Abs. 2 LV). Auch in diesem Falle sind die Abgeordneten an Aufträge und Weisungen nicht gebunden (Art. 11 Abs. 1 LV) das Urteil des LVerfG verpflichtet sie nicht, dem verfassungsändernden Gesetz zuzustimmen.

\section{5. d)}

Schließlich kann die Bestimmung eines Wahltermins in dem vom LVerfG gezogenen zeitlichen Rahmen auch durch eine ,Initiative aus dem Volk“ herbeigeführt werden. Wie sich aus Art. 42 Abs. 4 S. 2 LV ergibt, kann durch Volksentscheid auch ein verfassungsänderndes Gesetz beschlossen werden.

57 Zutreffend dargelegt bei Wissenschaftlicher Dienst des Landtags von Schleswig Holstein: Vermerk vom $08.092010,8 \mathrm{f}$.

58 Ebd., 10. 


\section{Fazit}

Das LVerfG hat mit seiner Entscheidung in der Sache LVerfG 1/10 seinerseits gegen das auch für das Gericht geltende Gebot der Verfassungsorgantreue verstoßen, indem es eine Verkürzung der laufenden Wahlperiode des Landtags angeordnet hat, ohne Landtag und Landesregierung in den Stand zu setzen, dieser Anordnung bei einem etwaigen Widerstand der Abgeordneten, die zu einem bestimmten Abstimmungsverhalten nicht gezwungen werden können, auch nachzukommen. Aus einem daraus sich möglicherweise ergebenden Verfassungskonflikt würden alle Verfassungsorgane beschädigt hervorgehen. Es bleibt nur der Appell an die Mitglieder des Landtags, jene verfassungspolitische Vernunft walten zu lassen, die das Urteil des LVerfG, abgesehen von seinen juristischen Mängeln, so schmerzlich vermissen lässt. 